I had therefore no hesitation, in view of the very unreliable knee joint which a ligamentous union of the patella would give him (he being dependent on his own exertions for a livelihood), in deciding to ligature the fragments with silver wire. This I did on November 29th, under the eucalyptus spray. The bone drill snapped in one of the fragments, and I had hard work to get it out again. I hammered the ends of the wire into the patella. After ligaturing, the wound was stitched up, covered with a good pad of dry boric lint and oil silk, and bandaged. No drainage tube was put in. The temperature the following day was $995^{\circ} \mathrm{F}$., and normal the day after, and continued so to the end. The wound was not undressed until Dec. 21st, twenty-two days after the operation, when the wound was found to be healed by first intention. Plaster-of-Paris bandages were then put on, and retained for three weeks. When removed, perfect union of the patella had taken place, and his leg was quite sound,
Some day I think of taking the wire out or nipping the end off it, as it irritates the skin over it.

Remarks.-This case is useful-1st, as showing the great benefits this operation confers, compared to the old plan of ligamentous union. With a weak foot on one leg and an unreliable knee on the other, this man would have been incapable of earning his living; 2 nd, as another added to the long list of successful ligatures of the patella for simple fracture. I was induced to send these two cases to you by the interesting nature of the "Occasional Records in the Science and Practice of Surgery," contributed to THE LANCET by Mr. Oliver Pemberton. The details of cases outside of hospital practice, treated by the operator himself throughout, with his reasons for such, may often very well be more interesting than the set notes by the surgical registrar or house-surgeon of another man's case.

Brisbane, Queensland.

\section{OSSEOUS UNION OF INTRA-CAPSULAR FRACTURE OF THE FEMUR.}

\section{Bx THOMAS F. RAVEN, L.R.C.P. \&c.}

A WOMAN, aged sixty-nine, slipped and fell, and on examination presented the usual symptoms of fracture of the neck of the femur, without impaction. She was treated with a long splint, and no extension was made. shaft. A short time ago she died, at the age of seventy-six, and I was enabled to secure the upper portion of each femur. The accompanying sketches, made with artistic fidelity by Mr. Arthur Trower, M.R.C.S., show how close to the head of the bone the fracture really was, and give a good idea of its complete bony union, and also of the contrast between the injured and uninjured bones, both in regard to the fracture itself and the resulting osteoarthritis.

Sir James Paget tells me, after an inspection of the bones, that he does not remember to have seen so thorough \&.

Anterior Aspect.

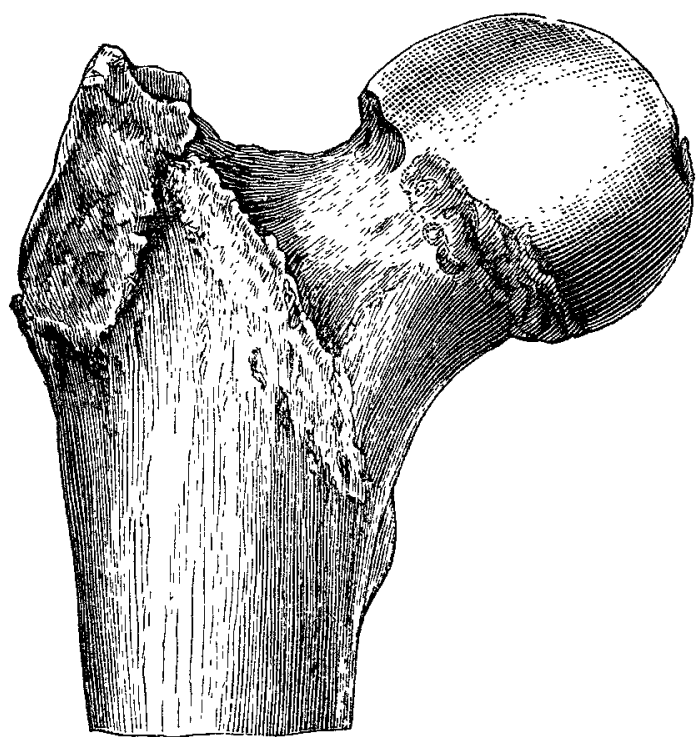

Right.

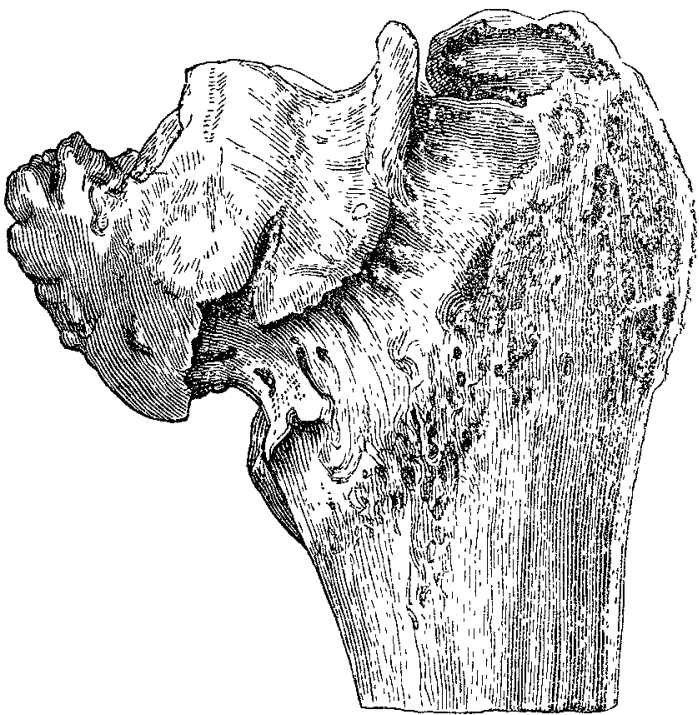

Left.

Bones removed from a woman, aged seventy-six years, who sustained a fracture of the neck of the left femur seven zears previous to her death. The sketch shows strong bony union and erosions of the head of the bone from arthritis.

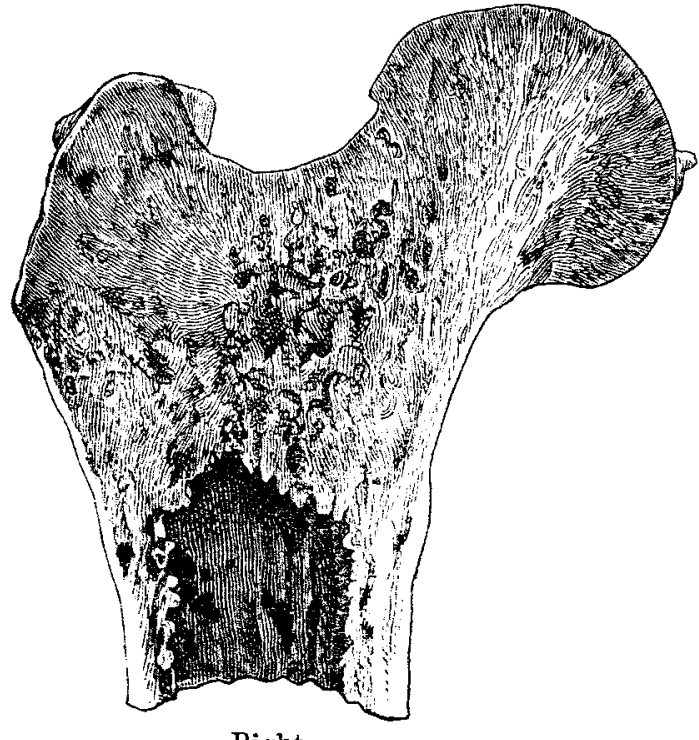

Right.

Sections.

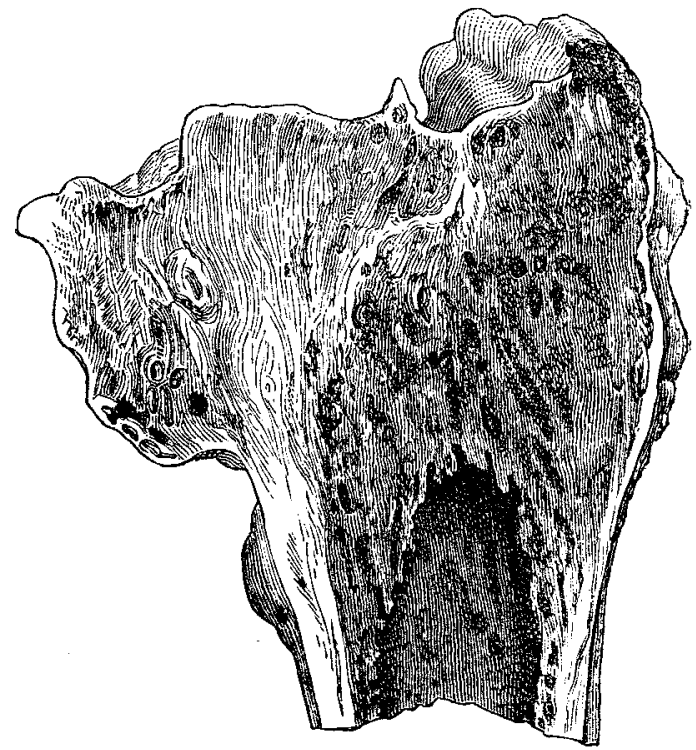

Left
Her recorery was so rapid and she walked so well subsequently, although with a shortened limb, that 'I was inclined to doubt the accuracy of my diagnosis, and to suspect that the fracture had occurred high up in the repair of this kind of fracture, and by his advice I hate presented the specimens to the Royal College of Surgeons, where they will be found in the Pathological Museum. Broadstairs. 